Article

\title{
Evaluating Olympic Pictograms Using Fuzzy TOPSIS - Focus on Judo, Taekwondo, Boxing, and Wrestling
}

\author{
Choi Kyoungho ${ }^{1}$, Kim Bong-seok ${ }^{2}$, Choi Jin-hee ${ }^{3 *}$ \\ 1 Department of Research Institute of Health Statistics/Radiological Science/, Jeonju University, Jeonju-si \\ 55069, Korea; ckh414@jj.ac.kr \\ 2 Department of Sports Coaching, Jeonju University, Jeonju-si 55069, Korea; kkkbbbq@jj.ac.kr \\ 3 Department of Fashion Business, Jeonju University, Jeonju-si 55069, Korea \\ * Correspondence: jhchoi@jj.ac.kr
}

Abstract: This study evaluated the ranking of comprehensibility of the pictograms for judo, taekwondo, boxing, and wrestling used in the six games from the 27th Sydney Olympics in 2000 to the 32nd Tokyo Olympics in 2021. The evaluation was done using the Fuzzy TOPSIS method, one of the multi-criteria decision-making methodologies commonly used in economics and others fields. The results are as follows. The first, pictograms from the 2008 Beijing Olympics ranked first in three sports: taekwondo, boxing, and wrestling, but there were no pictograms that consistently ranked first or sixth in all sports. Second, the result of the sensitivity analysis shows a possibility that the ranking will be reversed if the weight of the evaluation factors changes, but in the 1000time repetitive prediction, the better the evaluation ranking, the closer the value of the priority ranking to the ideal solution on average even if the weight changes.

Keywords: Olympic; pictogram; fuzzy; tosis

\section{Introduction}

The Olympics is an international event wherein thousands of athletes compete in several sporting events. Moreover, the Olympics, are held every two years, alternating between the Summer Olympics and Winter Olympics, under the aegis of the International Olympic Committee (IOC). The games originated from the ancient Greek Games held in Olympia, Greece from the 8th century BCE to the 5th century CE. The modern Olympics were revived by Pierre de Coubertin at the end of the 19th century, who founded the IOC in 1894, and two years later in 1896, the first modern Olympics were held in Athens, Greece [1].

Today, the Olympics have developed into an international sports and culture festival bringing people around the world together to uphold international amity, friendship, peace, and human culture, in a pure sporting spirit, transcending religion, race, and thought [2]. The Olympics have been held across the world; and in 1988 and 2018, South Korea hosted the 24th Summer Olympics and the 23rd Winter Olympics, respectively. The most recent 32nd Summer Olympics held in 2021 in Tokyo were postponed owing to the Covid-19.

The multinational attendance at the Olympics, transcends languages and cultures from various different countries and races. To facilitate smooth communication at such a large sporting event, the most representative nonverbal communication used are the Olympic pictograms. They first appeared at the 1936 Berlin Olympics in Germany, but became official at the 1964 Tokyo Olympics [3].

A pictogram is a compound image combining picture and telegram, that presents a message, and is used in international events for the ease of understanding and communication. According to American philosopher Peirce C. E., pictograms have the 
characteristics of an icon or picture. An icon is a symbol that uses a characteristic part of an object to be replaced by the object itself, while a symbol is in a fundamentally arbitrary relationship with the represented object [4]. Meanwhile, according to Bernard Marcel [5] symbols are related to meaning, while pictograms are related to the degree of description.

The function of a pictogram is 'to show the final destination into the process', 'to allow something', 'to warn something', 'to forbid something', and so on [6]. Meanwhile, the following conditions - 'easily identified', 'clear visualization', 'available and legible', 'clear graphic representation'- are presented as required for a pictogram. The Olympic pictograms are also used during the events; therefore, they must not be visually acceptable to people from various cultures, they must also be easy, quick, and accurate to understand by the general public.

Previous studies regarding the Olympic pictograms include Adir et al. [6-7] focusing on the production process of the Summer Olympics, and Akiyama [8] who focused on the accessibility of the Olympic and Paralympic pictograms. However, given the symbolism of the Olympic pictograms and their widespread usage, the studies are relatively few. Moreover, the standards and forms of pictograms used to express sport activities in accordance with the cultural characteristics of the host country are often unclear. Viewers often find it difficult to understand the actual sports represented in the pictogram. The Olympic pictograms, without such inconvenience, must satisfy the general conditions of universality, interest, and suitability.

However, few studies have evaluated whether the Olympic pictograms properly convey practical information to people. Therefore, it is necessary to evaluate whether pictograms related to Olympic sports viewed by people around the world are made to be accurately understood, thus fulfilling their functions and roles.

\section{Materials and Methods}

This study utilizes the Fuzzy TOPSIS technique (technique of order preference by similarity to ideal solution), a multi-criteria decision-making methodologies (MCDM) to rank the comprehensibility of pictograms for judo, taekwondo, boxing, and wrestling in the six Olympic games from the 27th Sydney Olympics in 2000 to the 32nd Tokyo Olympics in 2021. The findings of this study will help develop pictograms that can properly convey information on Olympic sports in the future. They can also be used and applied to develop pictograms in other sporting events, and thus enabling more people around the world to enjoy Olympic sports.

The main research problems of this study are as follows.

1. Research Question 1. Using the Fuzzy TOPSIS method, prioritizing the comprehensibility of the Olympic pictograms for judo, taekwondo, boxing, and wrestling events is to be evaluated;

2. Research Question 2. A sensitivity analysis is to be conducted on the TOPSIS results to explore the possibility of a change in priority resulting from changes in weight.

\subsection{Theoretical Reflection on the Methodology of Fuzzy TOPSIS}

Hwang and Yoon [9] proposed the TOPSIS methodology based in the linear ordering method proposed by Hellwig in 1968. Then, it was expanded into the fuzzy TOPSIS by Chen [10] using triangular fuzzy numbers in <Figure 1>. The theoretical background on fuzzy TOPSIS is well summarized in [11], [12], and so on. A brief summary of the fuzzy TOPSIS-based is laid out below

First, <Figure 2> presents the hierarchy of this study's decision-making problem. Next, form a decision-making matrix in the form of <Figure $3>$ composed of $n$ alternatives $A_{1}, A_{2}, \cdots, A_{n}$ and $m$, evaluation criteria $C_{1}, C_{2}, \cdots, C_{m}$. Here, $\widetilde{x_{l \jmath}}$ is the fuzzy value, $\widetilde{w_{J}}$ is the fuzzy weight of $m$ evaluation criteria. 
Definition. Triangular fuzzy number is a fuzzy number represented with three points as follows: $\mathrm{A}=\left(a_{1}, a_{2}, a_{3}\right)$. This representation is interpreted as membership functions.

$$
\mu_{A}(x)= \begin{cases}0 & \left(x<a_{1}\right) \\ \frac{x-a_{1}}{a_{2}-a_{1}} & \left(a_{1} \leq x \leq a_{2}\right) \\ \frac{a_{3}-x}{a_{3}-a_{2}} & \left(a_{2} \leq x \leq a_{3}\right) \\ 0 & \left(x \geq a_{3}\right)\end{cases}
$$

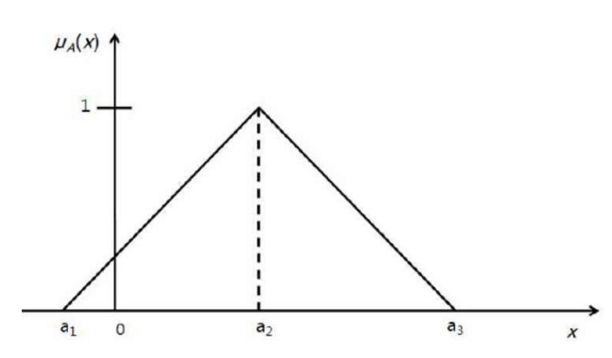

Figure 1. Triangular fuzzy numbers

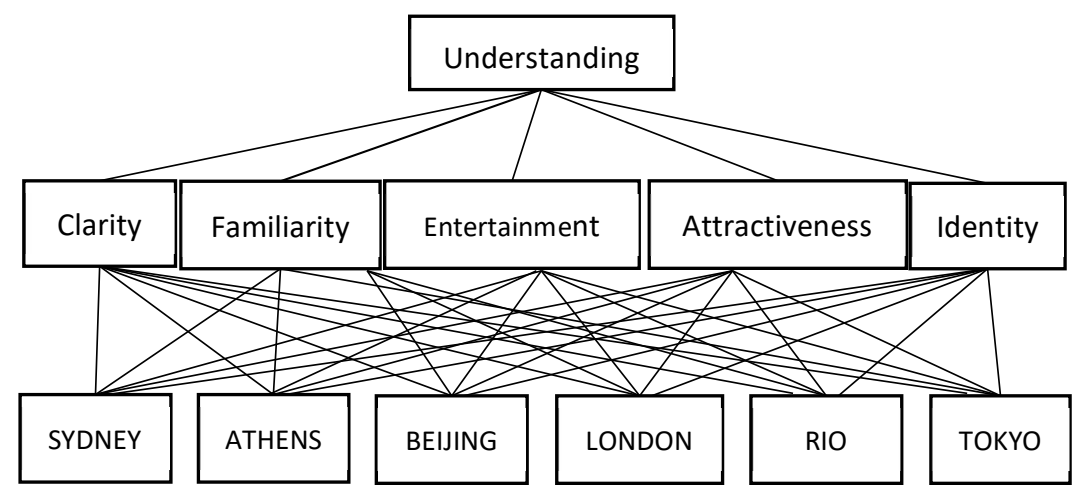

Figure 2. Hierarchy of the Decision-making Problem

\begin{tabular}{|c|c|c|c|c|c|}
\hline $\begin{array}{l}\text { Criterion } \\
\text { Alternative }\end{array}$ & $\begin{array}{c}\text { C1 } \\
\text { Clarity }\end{array}$ & $\begin{array}{c}\text { C2 } \\
\text { Clarity }\end{array}$ & $\begin{array}{c}\text { C13 } \\
\text { Clarity }\end{array}$ & $\begin{array}{c}\text { C4 } \\
\text { Clarity }\end{array}$ & $\begin{array}{c}\text { C5 } \\
\text { Clarity }\end{array}$ \\
\hline$A_{1}$ SYDNEY & $\widetilde{x_{11}}$ & $\widetilde{x_{12}}$ & $\widetilde{x_{13}}$ & $\widetilde{x_{14}}$ & $\widetilde{x_{15}}$ \\
\hline$A_{2}$ ATHENS & $\widetilde{x_{21}}$ & $\widetilde{x_{21}}$ & $\widetilde{x_{23}}$ & $\widetilde{x_{24}}$ & $\widetilde{x_{25}}$ \\
\hline$A_{3}$ BEIJING & $\widetilde{x_{31}}$ & $\widetilde{x_{32}}$ & $\widetilde{x_{33}}$ & $\widetilde{x_{34}}$ & $\widetilde{x_{35}}$ \\
\hline$A_{4} \mathrm{LONDON}$ & $\widetilde{x_{41}}$ & $\widetilde{x_{33}}$ & $\widetilde{x_{43}}$ & $\widetilde{x_{44}}$ & $\widetilde{x_{45}}$ \\
\hline$A_{5} \mathrm{RIO}$ & $\widetilde{x_{51}}$ & $\widetilde{x_{34}}$ & $\widetilde{x_{53}}$ & $\widetilde{x_{54}}$ & $\widetilde{x_{55}}$ \\
\hline$A_{6}$ TOKYO & $\widetilde{x_{61}}$ & $\widetilde{x_{35}}$ & $\widetilde{x_{63}}$ & $\widetilde{x_{64}}$ & $\widetilde{x_{65}}$ \\
\hline
\end{tabular}

Figure 3. Decision-making matrix

The process of selecting nearest alternatives to the fuzzy positive ideal solution (FPIS) and fuzzy negative ideal solution (FNIS) based on the principle of TOPSIS can be summarized into the following seven stages.

First stage: Calculate $\widetilde{x_{l j}}$, the fuzzy value, the constitutive element of the decisionmaking matrix in $<$ Figure $3>$ from the sample population.

Second stage: Calculate $\widetilde{w}_{J}$, the fuzzy weight of evaluation criteria from the respondents consisting of experts.

Third stage: Calculate the fuzzy weighted normalized matrix by normalizing $\widetilde{x_{\imath \jmath}}$ calculated in the first stage. 


$$
\widetilde{z}_{i j}=\frac{\tilde{x}_{i j}}{\sqrt{\sum_{i=1}^{n} \tilde{x}_{i j}^{2}}}, i=1, \ldots, n ; j=1, \ldots, m .
$$

Fourth stage: Calculate the weighted and normalized fuzzy value.

$$
\tilde{v}_{i j}=\tilde{w}_{j} \tilde{z}_{i j} \text {. }
$$

Fifth stage: Calculate FPIS and FNIS. Here, $J_{1}$ and $J_{2}$ implies the evaluation criteria for benefits and costs, respectively.

$$
\begin{aligned}
& \tilde{A}^{+}=\left\{\tilde{v}_{1}^{+}, \tilde{v}_{2}^{+}, \ldots, \tilde{v}_{m}^{+}\right\}=\left\{\left(\max _{i} \tilde{v}_{i j} \mid j \in J_{1}\right),\left(\min _{i} \tilde{v}_{i j} \mid j \in J_{2}\right) \mid i=1, \ldots, n\right\} \\
& \tilde{A}^{-}=\left\{\tilde{v}_{1}^{-}, \tilde{v}_{2}^{-}, \ldots, \tilde{v}_{m}^{-}\right\}=\left\{\left(\min _{i} \tilde{v}_{i j} \mid j \in J_{1}\right),\left(\max _{i} \tilde{v}_{i j} \mid j \in J_{2}\right) \mid i=1, \ldots, n\right\}
\end{aligned}
$$

Sixth stage: Calculate $d_{i}^{+}$, the Euclidean distance between each value at the fuzzy weighted and normalized matrix and FPIS as well as $d_{i}^{-}$, the Euclidean distance between each value and FNIS.

Seventh stage: Finally, find alternatives that are closest to the ideal solution and furthest from the negative solution. $\mathrm{CC}_{i}^{+}$has a value between 0 and 1 . The alternative with the greatest value is the optimal ideal.

$$
C C_{i}^{+}=\frac{d_{i}^{-}}{d_{i}^{+}+d_{i}^{-}}, \quad i=(1, \ldots, n)
$$

\section{Research Methodology}

\subsection{Research Flow Chart}

The flow chart of the process of this study is presented in $<$ Figure $4>$.

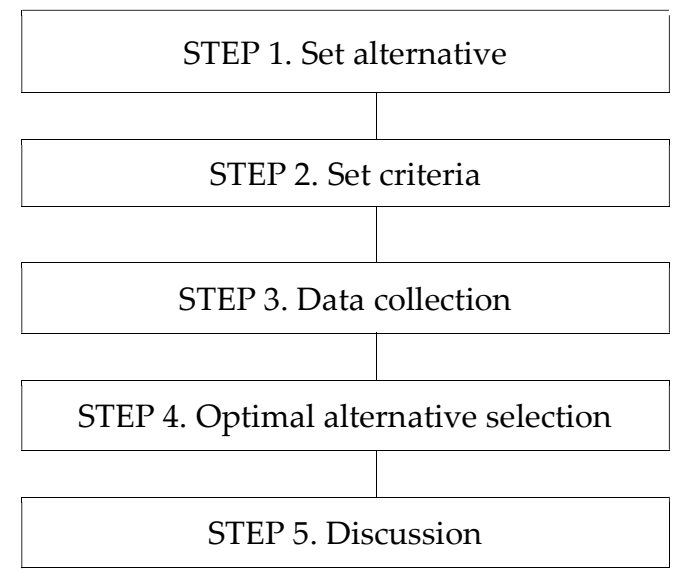

Figure 4. The process of this study.

\subsection{Setting Alternatives}

Establishing an alternative is the first task in designing a multi-criteria decisionmaking problem. Thus, an alternative should have viable and evaluable properties. In this study, the Olympic pictograms for judo, taekwondo, boxing, and wrestling presented in $<$ Table $1>$ were selected as alternatives for comprehensibility evaluation. 
Table 1. Pictogram of Judo, Boxing, Wrestling, Taekwondo at Olympic Games (2000 2020)

Criterion

\subsection{Determining the Evaluative Criteria}

In a multi-criteria decision-making problem, the evaluation criteria refer to those used in evaluating alternatives. There may be several evaluation criteria considered in the decision-making problem, but not all can be used. Furthermore, there is no absolute method of setting the evaluation criteria, but general criteria must be configured so that they do not overlap with each other or are skipped.

The evaluation criteria of this study were set as shown in $<$ Table $2>$, combining the general conditions that pictograms should possess (e.g., the function of delivering meaning easily and quickly) and the requirement of an infographic that delivers information using graphics [13]. Infographic is a compound word combining information and graphics as a method of information design, that transmits all kinds of verbal and visual messages through various media [14], [15].

Table 2. Evaluation Criteria for Comprehensibility of the Olympic Pictograms.

\begin{tabular}{cc}
\hline Evaluation Criterion & Meaning \\
\hline Clarity & The content of the information shall be easy, quick, and clear to \\
understand. \\
$\begin{array}{c}\text { Familiarity } \\
\text { Entertainment }\end{array}$ \\
$\begin{array}{c}\text { It shall visually stimulate curiosity and have a low repulsion. } \\
\text { Attractiveness }\end{array}$ \\
It shall be humorous and fun to approach. \\
Identity & It shall reflect the culture and traditions of the host country. \\
\hline
\end{tabular}

\subsection{Data Collection}

The value corresponding to each element of the decision-making matrix consisting of alternatives and evaluation criteria is the evaluation value of the alternative. This study thus, conducts sample selection by quarter random sampling the general public watching the Olympics divided by gender and age. The survey was conducted from May 10 to June 30 , 2021, wherein the 44 participants were asked to respond with a self-enumeration method. 
The size of experts selected for the calculation of weight in the Fuzzy TOPSIS was generally not large, considering it was 7 in Kabir Hasin [16] and 14 in Percin [17]. Therefore, seven experts, including three professors of fashion design, two professors of sports guidance, and two professors of industrial design, were surveyed for the weight determination conveying the importance of the five evaluation criteria selected in $<$ Table $2>$. For the survey for the comprehensibility evaluation and weight determination, linguistic ratings consisting of 5 levels were used as shown in $<$ Table $3>$.

Table 3. Linguistic ratings for alternative and weight

\begin{tabular}{cc}
\hline Alternative & Weight \\
\hline Very poor & Very low \\
Poor & Low \\
Fair & Medium \\
Good & High \\
Very good & Very high \\
\hline
\end{tabular}

Then, the measured values obtained by verbal evaluation were converted into fuzzy numbers according to Kore et al. [18] and presented in the range of 1 point to 9 points as shown in $<$ Table $4>$.

Table 4. Fuzzy ratings for linguistic ratings $t$

\begin{tabular}{ccc}
\hline Fuzzy number & Alternative & Weight \\
\hline$[1,1,3]$ & Very poor & Very low \\
{$[1,3,5]$} & Poor & Low \\
{$[3,5,7]$} & Fair & Medium \\
{$[5,7,9]$} & Good & High \\
{$[7,9,9]$} & Very good & Very high \\
\hline
\end{tabular}

\subsection{Analytical Tools}

This study utilizes two analytical tools. First, 'FuzzyTOPSISLiner' function from the 'FuzzyMCDM' package in the R-program [12]; [19] was used to resolve Research Question 1. Second, the PyTOPS program developed by Yadav et al. [20] with Python was used for the sensitivity analysis in TOPSIS presented in Research Question 2.

\subsection{Ethical Considerations}

Since data was collected for using the Fuzzy TOPSIS method was from humans, it was first reviewed by Jeonju University's institutional review board (IRB) (jjRB-210413HR-2021-0413). Coffee coupons were provided to respondents to express gratitude and to facilitate an environment where they could provide honest responses.

\section{Priority and Sensitivity Analysis}

\subsection{Evaluation of Priority Using Fuzzy TOPSIS}

The responses collected from seven experts to determine the weight of the five evaluation criteria presented in <Table $2>$ were integrated according to the fuzzy response synthesis algorithm presented in Kore et al. [18]. The result is as follows.

$$
\mathrm{W}=[(7,9,9),(1,5.857,9),(1,2.714,7),(3,7,9),(3,7,9)]
$$

A summary of the integrated fuzzy responses focusing on $a_{2}$, obtained from respondents consisting of men $(52.3 \%)$ and women $(47.7 \%)$, from age groups of 20 s or 
younger $(34.1 \%), 30$ s $(31.8 \%), 40$ s $(20.5 \%)$, and 50 s or older $(13.6 \%)$, is presented in Table 5.

Table 5. The integrated fuzzy responses

\begin{tabular}{|c|c|c|c|c|c|c|}
\hline Alternative & Criterion & Clarity & Familiarity & $\begin{array}{c}\text { Entertain } \\
\text { ment }\end{array}$ & $\begin{array}{c}\text { Attractiv } \\
\text { eness }\end{array}$ & Identity \\
\hline \multirow{6}{*}{ Judo } & SYDNEY & 4.818 & 4.954 & 5.136 & 4.863 & 5.227 \\
\hline & ATHENS & 7.636 & 7.090 & 6.045 & 6.500 & 5.818 \\
\hline & BEIJING & 5.590 & 5.090 & 5.363 & 4.772 & 5.545 \\
\hline & LONDON & 5.727 & 5.863 & 5.954 & 5.090 & 4.772 \\
\hline & RIO & 5.272 & 5.090 & 5.545 & 5.590 & 4.954 \\
\hline & TOKYO & 7.590 & 7.090 & 6.409 & 7.000 & 7.090 \\
\hline \multirow{6}{*}{$\begin{array}{c}\text { Taekwond } \\
\text { o }\end{array}$} & SYDNEY & 6.591 & 6.500 & 5.136 & 5.227 & 5.273 \\
\hline & ATHENS & 8.045 & 7.682 & 5.409 & 6.773 & 6.636 \\
\hline & BEIJING & 4.864 & 5.091 & 5.773 & 4.909 & 5.182 \\
\hline & LONDON & 5.818 & 5.455 & 6.773 & 6.136 & 5.227 \\
\hline & RIO & 5.591 & 5.318 & 5.818 & 5.318 & 4.090 \\
\hline & TOKYO & 5.318 & 5.273 & 4.909 & 4.273 & 5.000 \\
\hline \multirow{6}{*}{ Boxing } & SYDNEY & 3.909 & 3.500 & 4.500 & 4.000 & 4.273 \\
\hline & ATHENS & 6.818 & 5.818 & 5.545 & 5.182 & 5.591 \\
\hline & BEIJING & 5.318 & 5.091 & 5.818 & 4.955 & 5.500 \\
\hline & LONDON & 8.636 & 8.091 & 6.636 & 7.091 & 7.045 \\
\hline & RIO & 6.773 & 6.182 & 5.864 & 5.864 & 6.000 \\
\hline & TOKYO & 6.045 & 5.682 & 5.773 & 5.364 & 5.455 \\
\hline \multirow{6}{*}{ Wrestling } & SYDNEY & 2.272 & 2.500 & 3.681 & 3.318 & 3.227 \\
\hline & ATHENS & 3.681 & 3.818 & 5.045 & 4.636 & 4.590 \\
\hline & BEIJING & 4.454 & 4.636 & 5.272 & 4.863 & 5.363 \\
\hline & LONDON & 6.545 & 6.227 & 5.863 & 6.090 & 5.636 \\
\hline & RIO & 6.909 & 6.954 & 6.590 & 6.545 & 5.727 \\
\hline & TOKYO & 6.363 & 6.000 & 5.454 & 5.227 & 5.136 \\
\hline
\end{tabular}

Table 6 presents the result of priority ranking of the six alternatives by sports following the procedure to selecting the nearest alternatives to fuzzy positive ideal solution (FPIS) and fuzzy negative ideal solution (FNIS) based on the principle of TOPSIS. As part of this process, the 'FuzzyTOPSISLiner' function of the 'FuzzyMCDM' package in the R-program is used for the data and analysis program in $<$ Table $5>$ for the fuzzy response materials. There was no event that consistently ranked first or sixth across all sports. However, the pictograms of the 2008 Beijing Olympics ranked first for three sports including taekwondo, boxing, and wrestling. 
Table 6. Priority ranking of six alternatives by sports

\begin{tabular}{|c|c|c|c|c|}
\hline $\begin{array}{l}\text { Criterion } \\
\text { Ranking }\end{array}$ & Judo & Taekwondo & Boxing & Wrestling \\
\hline \multicolumn{5}{|l|}{1} \\
\hline & London & Beijing & Beijing & Beijing \\
\hline \multicolumn{5}{|l|}{2} \\
\hline & Rio & London & Tokyo & Athens \\
\hline \multicolumn{5}{|l|}{3} \\
\hline & Sydney & Rio & Rio & Tokyo \\
\hline \multicolumn{5}{|l|}{4} \\
\hline & Beijing & Tokyo & Athens & London \\
\hline \multicolumn{5}{|l|}{5} \\
\hline & Athens & Sydney & Sydney & Rio \\
\hline \multicolumn{5}{|l|}{6} \\
\hline & Tokyo & Athens & London & Sydney \\
\hline
\end{tabular}

\subsection{Sensitivity Analysis}

Sensitivity analysis was performed as a result of TOPSIS on the comprehensibility of the Olympic pictograms using Yadav et al. 's [19] PyTOPS program developed with Python. This was conducted to understand the possibility of a change in priority dependent on a change in the weights in the priority evaluation using the TOPSIS method. In other words, the result of the study is robust if the priority does not change significantly even when the weights for the five evaluation criteria used change. However, if the change in priority is high even with a small change in the weights, indicating a high sensitivity of the measurement results.

For sensitivity analysis, the changes in weights were $25 \%, 40 \%$, and $55 \%$. Within the given weight change, the simulation performed 1,000 times is presented in $\langle$ Table 7$\rangle$. It shows the means and standard deviations of the closeness to the ideal solution of top alternatives according to weight changes. $<$ Figure $1>$ is an example of judo, and it can be seen that the higher the ranking, the closer the average to the ideal solution even if the weights change. 
Table 7. Means and standard deviations of the closeness to the ideal solution of top alternatives

\begin{tabular}{|c|c|c|c|c|c|c|c|c|c|c|c|c|}
\hline \multirow{2}{*}{$\begin{array}{l}\text { Criterion } \\
\text { Ranking }\end{array}$} & \multicolumn{3}{|c|}{ Yudo } & \multicolumn{3}{|c|}{ Taekwondo } & \multicolumn{3}{|c|}{ Boxing } & \multicolumn{3}{|c|}{ Wrestling } \\
\hline & $25 \%$ & $40 \%$ & $55 \%$ & $25 \%$ & $40 \%$ & $55 \%$ & $25 \%$ & $40 \%$ & $55 \%$ & $25 \%$ & $40 \%$ & $55 \%$ \\
\hline 1 & $\begin{array}{c}0.989 \\
(0.002)\end{array}$ & $\begin{array}{c}0.916 \\
(0.021)\end{array}$ & $\begin{array}{c}0.989 \\
(0.002)\end{array}$ & $\begin{array}{c}0.916 \\
(0.013)\end{array}$ & $\begin{array}{c}0.916 \\
(0.021)\end{array}$ & $\begin{array}{c}0.916 \\
(0.028)\end{array}$ & $\begin{array}{c}1.000 \\
(0.000)\end{array}$ & $\begin{array}{c}1.000 \\
(0.000)\end{array}$ & $\begin{array}{c}1.000 \\
(0.000)\end{array}$ & $\begin{array}{c}1.000 \\
(0.000)\end{array}$ & $\begin{array}{c}1.000 \\
(0.000)\end{array}$ & $\begin{array}{r}1.000 \\
(0.000)\end{array}$ \\
\hline 3 & $\begin{array}{c}0.260 \\
(0.013)\end{array}$ & $\begin{array}{c}0.423 \\
(0.030)\end{array}$ & $\begin{array}{c}0.268 \\
(0.023)\end{array}$ & $\begin{array}{c}0.425 \\
(0.022)\end{array}$ & $\begin{array}{c}0.423 \\
(0.031)\end{array}$ & $\begin{array}{c}0.419 \\
(0.037)\end{array}$ & $\begin{array}{c}0.530 \\
(0.013)\end{array}$ & $\begin{array}{c}0.529 \\
(0.020)\end{array}$ & $\begin{array}{c}0.527 \\
(0.027)\end{array}$ & $\begin{array}{c}0.779 \\
(0.015)\end{array}$ & $\begin{array}{c}0.779 \\
(0.025)\end{array}$ & $\begin{array}{c}0.775 \\
(0.034)\end{array}$ \\
\hline 5 & $\begin{array}{c}0.195 \\
(0.012)\end{array}$ & $\begin{array}{c}0.210 \\
(0.024)\end{array}$ & $\begin{array}{c}0.148 \\
(0.021)\end{array}$ & $\begin{array}{c}0.212 \\
(0.016)\end{array}$ & $\begin{array}{c}0.209 \\
(0.023)\end{array}$ & $\begin{array}{c}0.208 \\
(0.032)\end{array}$ & $\begin{array}{c}0.335 \\
(0.006)\end{array}$ & $\begin{array}{c}0.335 \\
(0.010)\end{array}$ & $\begin{array}{c}0.336 \\
(0.014)\end{array}$ & $\begin{array}{c}0.351 \\
(0.010)\end{array}$ & $\begin{array}{c}0.352 \\
(0.016)\end{array}$ & $\begin{array}{c}0.354 \\
(0.022)\end{array}$ \\
\hline 6 & $\begin{array}{c}0.089 \\
(0.011)\end{array}$ & $\begin{array}{c}0.181 \\
(0.020)\end{array}$ & $\begin{array}{c}0.087 \\
(0.023)\end{array}$ & $\begin{array}{c}0.184 \\
(0.014)\end{array}$ & $\begin{array}{c}0.179 \\
(0.021)\end{array}$ & $\begin{array}{c}0.174 \\
(0.026)\end{array}$ & $\begin{array}{r}0.000 \\
(0.000)\end{array}$ & $\begin{array}{c}0.000 \\
(0.000)\end{array}$ & $\begin{array}{c}0.000 \\
(0.000)\end{array}$ & $\begin{array}{c}0.000 \\
(0.000)\end{array}$ & $\begin{array}{c}0.000 \\
(0.000)\end{array}$ & $\begin{array}{c}0.000 \\
(0.000)\end{array}$ \\
\hline
\end{tabular}

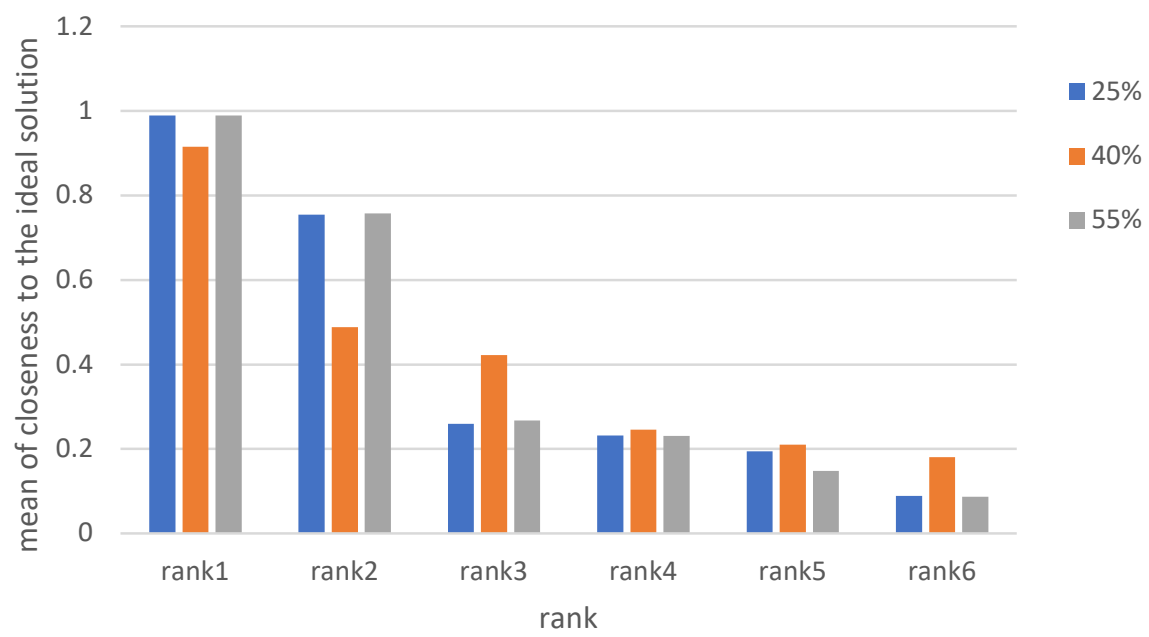

Figure 5. Means of the closeness to the ideal solution of top alternatives (exam: Judo)

\section{Results and discussion}

The Olympics are not just sporting events; they have developed into a great festival where all participating nations come together as a community comprising of different religions and cultures . Furthermore, the Olympic Games provide prime opportunity for the host countries to promote their culture and traditions to the world and to provide economic profits generating and national development opportunities. In other words, hosting the Olympics is a step forward for many countries.

As such, the host country usually strives to promote and communicate its Olympics to the world in various ways. A typical method is the use of a pictogram that started with the 1964 Tokyo Olympics. Ease of communication is most important function of pictograms in the Olympics. It is thus, undesirable to have pictograms representing specific events, which are difficult to understand owing to cultural characteristics unique 
to the host country. Therefore, Olympic pictograms should satisfy the general conditions of pictograms such as universality, interest, and suitability; at the same time, they should be evaluated from an academic point of view.

This study evaluated the ranking of comprehensibility of the pictograms for judo, taekwondo, boxing, and wrestling used in the six games from the 27th Sydney Olympics in 2000 to the 32nd Tokyo Olympics in 2021. The evaluation was done using the Fuzzy TOPSIS method, one of the multi-criteria decision-making methodologies commonly used in economics and others fields. The results are as follows.

First, the pictograms from the 2008 Beijing Olympics ranked first in three sports: taekwondo, boxing, and wrestling, but there were no pictograms that consistently ranked first or sixth in all sports. Nonetheless, the pictogram, which was evaluated in the sixth place, showed low clarity.

Second, the result of the sensitivity analysis shows a possibility that the ranking will be reversed if the weight of the evaluation factors changes, but in the 1000-time repetitive prediction, the better the evaluation ranking, the closer the value of the priority ranking to the ideal solution on average even if the weight changes. Paradoxically, such a result implies that incorrect weighting of the evaluation factors may lead to an incorrect evaluation, suggesting the importance of the former. Furthermore, each evaluation factor can have a significant impact on the evaluation results.

\section{Conclusions}

In this study, five evaluation factors consisting of clarity, familiarity, entertainment, attractiveness, and identity were considered, based on the conditions that infographics and pictograms must satisfy. However, there may be evaluation factors that may be considered other than these five factors in evaluating the Olympic pictograms; thus, further research is needed. Raising the need to evaluate the performance of Olympic pictograms and suggesting evaluation methods, this study results aid in developing pictograms, as a means of promoting Olympic sports in the future.

Author Contributions: Conceptualization, methodology, and formal analysis, K.C.; investigation, s resources, B. C; writing-review and editing, and visualization, J.C. All authors have read and agreed to the published version of the manuscript.

Funding: This research received no external funding.

Institutional Review Board Statement: The study was conducted according to the guidelines of the Declaration of Helsinki, and approved by the Institutional Ethics Committee of Jeonju University((jjRB-210413-HR-2021-0413).

Informed Consent Statement: Informed consent was obtained from all subjects involved in the study. Written informed consent has been obtained from the patient(s) to publish this paper.

Data Availability Statement: The data presented in the study are available on request from the corresponding authors.

Conflicts of Interest: The authors declare no conflict of interest.

\section{References}

1. Wikipedia. https://ko.wikipedia.org/wiki/

2. Park, S. Y. (2014). Study on the recognition and preference of Olympic pictogram design, Journal of Digital Design, 14(4), 687696.

3. Kim, K. S., Um, G. J. (2019). A study on the expression of Olympic pictogram symbols by national identity, Korea Institute of Design Research Society, 4(3), 52-62.

4. Park, J. (2005). A study on seeing mechanism of decoding pictograms and international standardization, Journal of Korean Society of Design Science, 18(2), 345-354. 
5. Bernard, P., Marcel, T. (1984). Representation and understanding in the use of symbols and pictogram, Information Design, 3775, Colchester, John Wiley and Sons.

6. Adir, V. Adir, G., Dobrescu, T. (2015). How to achieve a graphic language in a signage system, Journal of Industrial Design and Engineering Graphics, 10, 5-8.

7. Adir, V., Pascu, N. E., Adir, G., Ivascu, L. (2017). The meaning of pictogram in a graphic and visual language, Journal of Industrial Design and Engineering Graphics, 12(2), 39-43.

8. Akiyama, T. (2020). Olympic and Paralympic games and legacy of pictogram and accessibility, Journal of Japan Society of Civil Engineers, 105(7), 22-25.

9. Hwang, C. L., Yoon, K. (1981). Multiple attribute decision making, Methods and Applications, Springer, Berlin.

10. Chen, C. T. (2000). Extensions of the TOPSIS for group decision-making under fuzzy environment, Fuzzy Sets and Systems, 114, 1-9.

11. Salabun, W. J. (2015). The characteristic objects method: A new distance-based approach to multicriteria decision-making problems, Journal of multi-Criteria Decision Analysis, 22, 37-50.

12. Dudek, A., Jefmanski, B. (2015). The fuzzy topsis method and implementation in the R program, Business Informatics, 35(1), 19-27.

13. Kwon, H. J. (2013). Elements for evaluating the usability of the wed-based inforgraphic design, Journal of Korea Multimedia Society, 16(7), 879-887.

14. Pettersson, R. (1998). What is information design? Vision Plus 4, Proceedings of International Institute for Information Design, 58-74.

15. Noh, S. M., Son, J. (2014). An analysis of the inforgraphics features of visualization materials in section 'information and communication' of Physics I textbook, Journal of the Korean Association for Science Education, 34(4), 359-366.

16. Kabir, G., Hasin, M. A. A. (2012). Comparative analysis of topsis and fuzzy topsis for the evaluation of travel website service quality, International Journal for Quality Research, 6(3), 169-185.

17. Percin, S. (2019). A combined fuzzy multicriteria decision-making approach for evaluating hospital website quality, Journal of Multi-Criteria Decision Analysis, 26, 129-144.

18. Kore, N. B., Ravi, K., Patil, S. B. (2017). A simplified description of fuzzy topsis method for multi criteria decision making, International Research Journal of Engineering and Technology, 4(5), 1-4

19. Comprehensive R Archive Network. https://cran.r-project.org/web/packages/FuzzyMCDM/index.html

20. Yadav, V., Karmakar, S., Kalbar, P. P., Dikshit, A. K. (2019). PyTOPS: A python based tool for topsis, SoftwareX, 9, $217-222$. 